\title{
Anticollision Method of Active Magnetic Guidance Ranging for Cluster Wells
}

\author{
Xinyu Dou $\mathbb{D}^{1,2,3}$ Huaqing Liang $\mathbb{D}^{1,2}$ and Yang Liu ${ }^{1,2}$ \\ ${ }^{1}$ State Key Laboratory of Petroleum Resources and Prospecting, China University of Petroleum-Beijing, Beijing 102249, China \\ ${ }^{2}$ College of Geophysics and Information Engineering, China University of Petroleum-Beijing, Beijing 102249, China \\ ${ }^{3}$ Intelligence and Information Engineering College, Tangshan University, Tangshan 063000, China
}

Correspondence should be addressed to Huaqing Liang; hqliang@cup.edu.cn

Received 6 February 2018; Revised 24 March 2018; Accepted 11 April 2018; Published 15 May 2018

Academic Editor: Milica Rančić

Copyright (C) 2018 Xinyu Dou et al. This is an open access article distributed under the Creative Commons Attribution License, which permits unrestricted use, distribution, and reproduction in any medium, provided the original work is properly cited.

\begin{abstract}
Conventional adjacent wells range scanning calculations cannot meet the accuracy demands of the anticollision measurement of borehole distances any longer. Current techniques commonly employ electromagnetic detection tools while drilling; this requires putting equipment down adjacent wells to avoid collision risks, which adds more workload and costs and sometimes even affects the normal production of the producing wells. Measuring and tracking adjacent borehole distances while drilling is an essential process that guides the drill bit and effectively avoids collisions with existing wells. This paper proposes an active anticollision method of rotating magnetic ranging based on double symmetrical magnetic sources with opposite magnetic moments. First, the proposed method uses magnetic sources in the drilling well that are built into the probe tube to generate a magnetic field; then, the ferromagnetic casing of the existing well would be magnetized by the abovementioned magnetic field; finally, the magnetization field of the ferromagnetic casing is measured by a triaxial magnetometer built into the probe tube to determine the spacing and position of the existing well. Simultaneously, the calculation models of magnetic flux density around the casing of the existing well and magnetic sources are established, the calculation formulae of the relative distance and position of two adjacent wells are deduced, and a new variable interval section segmentation is proposed based on the Cosine theorem. The simulation results demonstrate that the spacing and position of the existing well are determined based on the magnetic sources' spacing inside the probe, the magnetic moment of the magnetic sources, the relative permeability of the casing, the diameter of the casing, and the inclination between the drilling well and the existing well. The validity and accuracy of the active magnetization model are confirmed, providing theoretical support for the further development of electromagnetic anticollision devices.
\end{abstract}

\section{Introduction}

Complex wells can effectively improve single well productivity and the ultimate recovery of complex oil and gas fields; magnetic guidance technology is the core technology of drilling engineering in complex structure wells [1]. The working principle of the anticollision scanning method [2] is fitting and approximating the real drilling trajectory with inclinometer data; then, the relative position relationship between the drilling well and the existing well can be calculated by the anticollision scanning algorithm. When the relative borehole distance is less than the given safety distance, the drilling direction can be adjusted to avoid a collision accident. The accuracy of the anticollision scanning method relies on inclinometer data of borehole trajectories for the drilling well and the existing well, borehole trajectory fitting approximation method, and the anticollision scanning algorithm. These data and algorithms do tend to have errors in practical applications; the cumulative errors increase with increased drilling depth, causing the accuracy of the calculation results to be lower. Therefore, this method fails to effectively avoid wellbore collisions. To better solve this problem, it is necessary to monitor the distance between adjacent wells and maintain it within a reasonable range in real time. The newly emerged electromagnetic guidance tools can guarantee safe drilling and the successful avoidance of collisions.

Electromagnetic guidance tools, which include the magnetic guidance tool (MGT) [3], the Rotating Magnet Ranging 
Service (RMRS) system [4], the Wellspot Tool (WT) [5], and the Single Wire Guidance (SWG) [6] tool, can detect the distance between the drilling well and the existing well to avoid collision [1-6]. MGT and RMRS are applied in adjacent twin parallel Steam Assisted Gravity Drainage (SAGD) wells that guide the drilling of an injection well to ensure that it is parallel to the production well $[2,3,7-9]$. However, it requires improvement if it is to be applied to the anticollision of cluster wells. There are two primary disadvantages of the MGT and RMRS methods. First, they are difficult to operate, as a probe tube must be placed in the existing well to guide the detection of drill bit. Second, the monitoring workload of the anticollision system is quite heavy. WT is not suitable for the anticollision of cluster wells since the detection process requires pulling out the drilling tools. The SWG tool overcomes the disadvantages of the MGT and RMRS $[1,4]$, namely, that neither reduces the workloads, nor requires pulling out the drilling tool to guide the detection device. However, there are three primary disadvantages of the SWG tool. First, a cable must be placed down the offset well, which affects the normal production process of the existing well. Additionally, a large amount of equipment must be utilized in order to run this system. Lastly, the technology of the SWG tool is monopolized by foreign companies and the service cost is expensive, so domestic companies rarely use SWG to reduce the cost of a single well.

With cluster wells being used in more and more applications in land and sea environments, the borehole distance has been reduced from $2 \mathrm{~m} \times 2 \mathrm{~m}$ to $1.5 \mathrm{~m} \times 1.7 \mathrm{~m} \mathrm{[2,4]}$, and infilling adjustment wells are usually added to the existing well pattern. The traditional anticollision scanning method is insufficient for cluster wells' anticollision. Therefore, the active magnetic guidance ranging method is proposed to avoid borehole collision. This method is based on detecting magnetic induced intensity of the casing in the existing well; it detects the borehole distance during the drilling process in real time without interrupting drilling or ever requiring any other equipment to be placed in the existing well. The method becomes practically significant in providing a theoretical basis for the research and development of active anticollision devices for cluster wells.

\section{Principle of Active Magnetic Guidance Ranging}

The signal sources or the probe tube must be mounted in the existing well to measure the distance between drilling well and offset wells for MGT, RMRS, SWG, and other electromagnetic guidance tools [10-13]. To solve the above problems, the active magnetic anticollision ranging method of cluster wells is proposed based on the fact that the magnetic poles of double magnetic sources are parallel to each other with opposite magnetic moments (expressed as red and green lines in Figure 1). It does not interrupt the drilling of the production well, nor does it require any other equipment to be placed in the offset well, yet it detects the borehole distance in real time during the drilling process. This principle is shown in Figure 1.

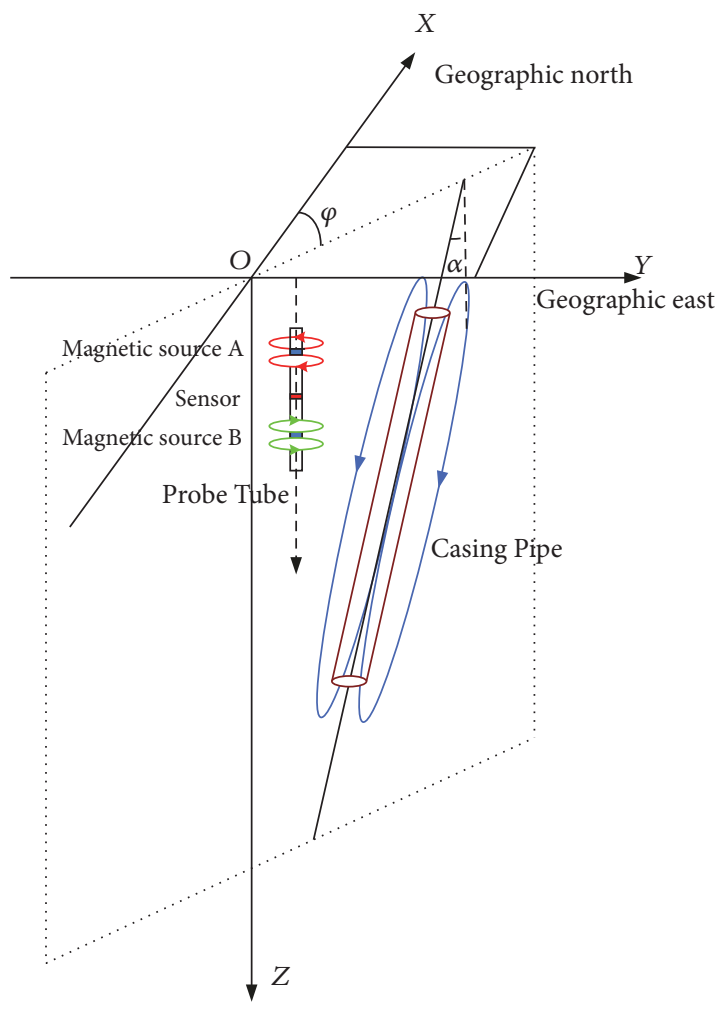

FIGURE 1: Principle of active magnetic guidance ranging for cluster wells.

Therefore, the total magnetic induction intensity as detected by a triaxial magnetometer is 0 when there is no casing around the probe tube or when the casing is far away from the probe tube [14]. When there is casing around the probe tube, the triaxial magnetometer data represent the sum of the geomagnetic field, the magnetic field of two magnetic sources, and the magnetic flux density of the magnetizing casing which generates a magnetic field along its axial direction (expressed as blue lines). As the magnetic fields of magnetic sources vary with the rotation of the drill rod, the alternating magnetic field frequency of the casing varies along with the rotation rate of magnetic sources.

Based on the above described principle, the relative distance and orientation between the drilling well and the existing well are calculated using the detection data of a triaxial magnetometer, and the well trajectory of the drilling well is adjusted appropriately to prevent collisions with the existing well.

\section{Calculation Method of Adjacent Well Distance}

The calculation method of adjacent well distance is the anticollision core technology of magnetic guidance. Under the coordinate system shown in Figure 1, the casing stretches vertically downward along the $Z$-axis, and the $X$-axis points to geographic north. The outer diameter and thickness of the 


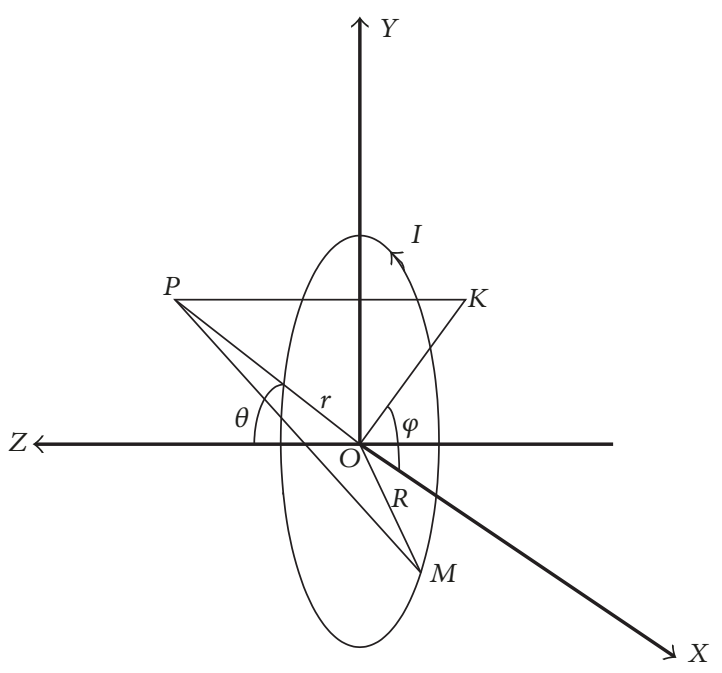

FIgURE 2: Schematic diagram of magnetic dipole.

casing are $C$ and $\delta$, respectively. The inclination and azimuth of the casing are $\alpha$ and $\varphi$, respectively.

\subsection{Distribution of Magnetic Field around Magnetic Sources.} The dimensions of the components are strictly limited by the geometrical size of the probe tube in the drilling well. The two symmetrical magnetic sources' sizes must be far less than the size of drill collar, and the mud flow channel should be reserved. The magnetic source is a cylinder with a length of $100 \sim 200 \mathrm{~mm}$ and a diameter of $20 \sim 50 \mathrm{~mm}$ according to common drill collar parameters. To ensure an anticollision system for cluster well applications, the borehole distance should be at least $1 \mathrm{~m}$ and normally more than $3 \mathrm{~m}$; otherwise, the probability of collision is increased. The size of the magnetic source is much smaller than the adjacent borehole distances; thus, the magnetic source can be regarded as a magnetic dipole $[1,15,16]$.

The spherical coordinate system is established as shown in Figure 2. The magnetic dipole, modeled with a circular current loop, is placed in the XOY plane. The spherical coordinate system can be established by taking the center of the magnetic dipole as the origin point and the direction of the magnetic moment of the magnetic dipole as the $Z$-axis. The spherical coordinates of point $P$, that is, any point in space, can be expressed as $(r, \theta, \varphi)$. The vector distance from point $P$ to point $O$ is $r$, the intersection angle between $P O$ and the $Z$-axis is $\theta$, and the angle between the $X$-axis and $K O$, which is the projection of $P O$ on the $X Y$-plane, is $\varphi$. Expression (1) gives the relations between the spherical and Cartesian coordinates [17].

$$
\begin{aligned}
& r=\frac{x}{\sin \theta \cos \varphi}=\frac{y}{\sin \theta \sin \varphi}=\frac{z}{\cos \theta}, \\
& \theta=\arctan \left(\frac{\sqrt{x^{2}+y^{2}}}{z}\right), \varphi=\arctan \left(\frac{y}{x}\right) .
\end{aligned}
$$

Assuming that point $M$ is any point on the circle, its corresponding coordinate is $\left(r, \pi / 2, \varphi_{1}\right)$, and the magnetic flux density caused by the current unit $I d \mathbf{l}$ in the space point $P$ can be calculated by the Biot-Savart Law as follows:

$$
d \mathbf{B}=\frac{\mu}{4 \pi} \frac{I d \mathbf{l} \times \mathbf{a}}{a^{3}},
$$

where $\mathbf{a}, d \mathbf{l}$, and $\mu$ are the distance vector of point $M$ to point $P$, the tangent vector of point $M$, and the space magnetic permeability, respectively. In the spherical coordinate system, those parameters have the following forms:

$$
\begin{aligned}
\mathbf{a}= & \left(r \sin \theta \cos \varphi-R \cos \varphi_{1}\right) \vec{i} \\
& +\left(r \sin \theta \sin \varphi-R \sin \varphi_{1}\right) \vec{j}+(r \cos \varphi) \vec{k}, \\
d \mathbf{l}= & (d x, d y, d z)=(-R \sin \varphi d \varphi, R \cos \varphi d \varphi, 0), \\
\frac{1}{a^{3}}= & \frac{1}{\left(\sqrt{r^{2}+R^{2}}\right)^{3}}\left[1-\frac{2 r R \sin \theta}{r^{2}+R^{2}} \cos \left(\varphi-\varphi_{1}\right)\right]^{-3 / 2}, \\
m= & I S=\pi I R^{2} .
\end{aligned}
$$

In previous expressions with $R$ the radius of the loop is denoted and $m$ is the magnetic dipole moment. By performing the integral calculation and substituting Expressions (3) in formula (2), when far from the loop, the above formula can be expressed as

$$
\begin{aligned}
& B_{x}=\frac{3 \mu}{8 \pi} \frac{m}{\left(\sqrt{r^{2}+R^{2}}\right)^{3}} \frac{r^{2} \sin 2 \theta \cos \varphi}{r^{2}+R^{2}}, \\
& B_{y}=\frac{3 \mu}{8 \pi} \frac{m}{\left(\sqrt{r^{2}+R^{2}}\right)^{3}} \frac{r^{2} \sin 2 \theta \sin \varphi}{r^{2}+R^{2}}, \\
& B_{z}=\frac{\mu}{2 \pi} \frac{m}{\left(\sqrt{r^{2}+R^{2}}\right)^{3}}\left(1-\frac{3 r^{2} \sin ^{2} \theta}{2\left(r^{2}+R^{2}\right)}\right) .
\end{aligned}
$$

The detecting data of the magnetic anticollision system with a rotating probe tube which is driven by the drill string are collected for the cluster well while drilling. Put simply, the magnetic dipole rotates around the $Y$-axis, and the distance $r$ between the casing and the magnetic dipole can be regarded as a constant, $\varphi=0,0 \leq \theta \leq 2 \pi$.

$$
\begin{aligned}
& B_{x}=\frac{3 \mu}{8 \pi} \frac{m}{\left(\sqrt{r^{2}+R^{2}}\right)^{3}} \frac{r^{2} \sin 2 \theta}{r^{2}+R^{2}}, \\
& B_{y}=0, \\
& B_{z}=\frac{\mu}{2 \pi} \frac{m}{\left(\sqrt{r^{2}+R^{2}}\right)^{3}}\left(1-\frac{3 r^{2} \sin ^{2} \theta}{2\left(r^{2}+R^{2}\right)}\right) .
\end{aligned}
$$

If the distance from any point $P$ on the casing to the magnetic source is much larger than the radius of the magnetic source $(r \gg R)$, then

$$
B=\sqrt{B_{x}^{2}+B_{y}^{2}+B_{z}^{2}}=\frac{\mu m}{2 \pi r^{3}} \sqrt{1-\frac{3}{4} \sin ^{2} \theta} .
$$




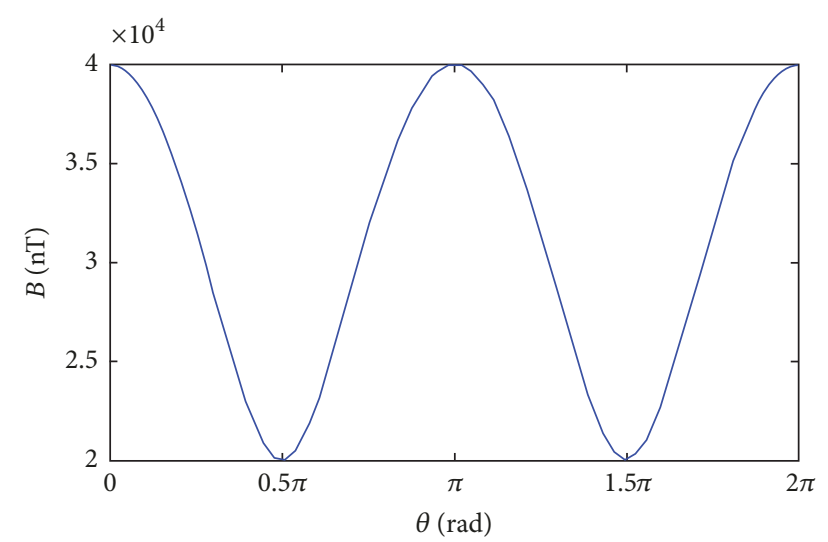

Figure 3: Magnetic flux density simulation.

By assuming $\mu=4 \pi \times 10^{-7} \mathrm{H} / \mathrm{m}, m=200 \mathrm{~A} \cdot \mathrm{m}^{2}, r=1 \mathrm{~m}$, $\theta \in[0,2 \pi]$, the curve of magnetic flux density $B$ is shown in Figure 3.

During the rotation of the probe from Figure 3, the axial direction of the magnetic source points directly to the casing whenever the maximum magnetic flux density of casing reaches one of the corresponding coordinates, namely, $0,0.5 \pi, \pi, 1.5 \pi$, and $2 \pi$. The direction of the casing can be determined by the maximum magnetic flux density. The distance from the probe tube to the casing can be calculated by using the relationship between the relative distance and the magnetic flux density of the magnetizing casing. Therefore, the maximum and minimum values on the curve indicate that the axis of the magnetic source is directly pointing to the casing at these points. At the moment the curve reaches its peak or valley, the azimuth of the casing in the adjacent well can be determined by measuring the magnetic azimuth of the probe tube with the built-in triaxial magnetometer.

3.2. Magnetizing Field Calculation of Casing. Due to the complexity of the actual formation, the following five assumptions are proposed to simplify the calculation [18-20]:

(1) The formation is evenly distributed and isotropic.

(2) The casing length is infinite.

(3) The radius of the casing is much less than the distance between the drilling well and the existing well.

(4) The casing is isotropic.

(5) There are no ferromagnetic minerals with high permeability in the formation.

Under these conditions, the magnetic flux density of the casing in the existing well can be calculated by formula (4).

\subsubsection{Magnetic Flux Density Calculation of the Magnetizing} Casing for the Upper Magnetic Source. Taking the upper magnetic source center of the probe tube as the origin $C$, the $Y$-axis and $Z$-axis correspond to the axial directions of the probe tube and the upper magnetic source, respectively. The coordinate system is established in Figure 4 . The distance $O E$ from the probe tube center point $O$ to the casing is $d$, the intersection angle between the axes of the casing and the probe tube is $\alpha$, the distance between the two magnetic

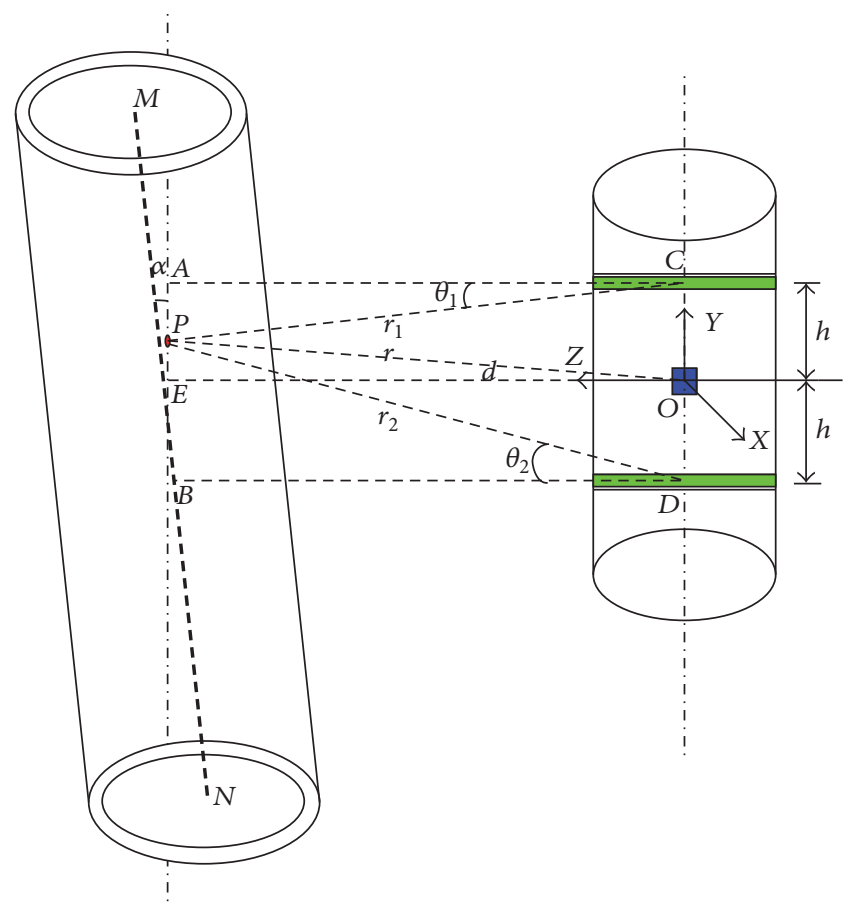

FIgURE 4: Calculation model of magnetic intensity in the magnetized casing.

sources is $2 h$, the angle between $P C$ and the upper magnetic source axis is $\theta_{1}$, the distance from point $P$ to point $C$ is $r_{1}$, and point $P$ is any point on the casing. The permeability of the casing is $\mu_{1}=\mu_{0}\left(1+\chi_{m}\right)$, the magnetic susceptibility of the casing is $\chi_{m}$, and the vacuum permeability is $\mu_{0}$.

The magnetic flux density distribution around the upper magnetic source can be deduced by formula (4) as follows:

$$
\begin{aligned}
& B_{C x}=\frac{3 \mu}{8 \pi} \frac{m}{r_{1}^{3}} \sin 2 \theta_{1} \cos \varphi, \\
& B_{C y}=\frac{3 \mu}{8 \pi} \frac{m}{r_{1}^{3}} \sin 2 \theta_{1} \sin \varphi, \\
& B_{C z}=\frac{\mu}{2 \pi} \frac{m}{r_{1}^{3}}\left(1-\frac{3}{2} \sin ^{2} \theta_{1}\right),
\end{aligned}
$$

where $\mu$ is the permeability of the space surrounding the magnetic source.

From the previous analysis, the magnetic flux density of the magnetizing casing reaches maximum when the magnetic source axis points to the casing. Therefore, the magnetic flux density distribution around the magnetic source can be deduced as follows:

$$
\begin{aligned}
& B_{C x}=0 \\
& B_{C y}=\frac{3 \mu m}{8 \pi r_{1}^{3}} \sin 2 \theta_{1} \\
& B_{C z}=\frac{\mu m}{2 \pi r_{1}^{3}}\left(1-\frac{3}{2} \sin ^{2} \theta_{1}\right) .
\end{aligned}
$$


For the upper magnetic source, the magnetic flux density of the magnetizing casing can be expressed as follows:

$$
\begin{aligned}
& B_{C x}^{\prime}=0, \\
& B_{C y}^{\prime}=\frac{3 \mu_{1} m}{8 \pi r_{1}^{3}} \sin 2 \theta_{1}, \\
& B_{C z}^{\prime}=\frac{\mu_{1} m}{2 \pi r_{1}^{3}}\left(1-\frac{3}{2} \sin ^{2} \theta_{1}\right) .
\end{aligned}
$$

3.2.2. Magnetic Flux Density Calculation of the Magnetizing Casing for the Lower Magnetic Source. Similar to formula (7), for the lower magnetic source built into the probe tube, the magnetic flux density of the magnetizing casing can be expressed as follows:

$$
\begin{aligned}
& B_{D x}^{\prime}=0, \\
& B_{D y}^{\prime}=-\frac{3 \mu_{1} m}{8 \pi r_{2}^{3}} \sin 2 \theta_{2}, \\
& B_{D z}^{\prime}=-\frac{\mu_{1} m}{2 \pi r_{2}^{3}}\left(1-\frac{3}{2} \sin ^{2} \theta_{2}\right) .
\end{aligned}
$$

3.2.3. Total Magnetic Flux Density Calculation of the Casing. The formulae of the magnetic flux density of the magnetizing casing caused by the two different magnetic sources are derived below.

The magnetic flux density caused by the upper magnetic source can be calculated using the following:

$$
\begin{aligned}
& B_{P C x}=\frac{3 \mu_{1}}{8 \pi} \frac{m}{r_{1}^{3}} \sin 2 \theta_{1} \cos \varphi, \\
& B_{P C y}=\frac{3 \mu_{1}}{8 \pi} \frac{m}{r_{1}^{3}} \sin 2 \theta_{1} \sin \varphi, \\
& B_{P C z}=\frac{\mu_{1}}{2 \pi} \frac{m}{r_{1}^{3}}\left(1-\frac{3}{2} \sin ^{2} \theta_{1}\right) .
\end{aligned}
$$

$$
\begin{aligned}
& B_{P x}=\frac{3 \mu_{1} m x}{4 \pi \sqrt{x^{2}+y^{2}}}\left[\frac{\sqrt{(y-h)^{2}+x^{2}}\left(\sqrt{d^{2}-x^{2}}+y \tan \alpha\right)}{\left(\sqrt{(y-h)^{2}+x^{2}+\left(\sqrt{d^{2}-x^{2}}+y \tan \alpha\right)^{2}}\right)^{5}}-\frac{\sqrt{(y+h)^{2}+x^{2}}\left(\sqrt{d^{2}-x^{2}}+y \tan \alpha\right)}{\left(\sqrt{(y+h)^{2}+x^{2}+\left(\sqrt{d^{2}-x^{2}}+y \tan \alpha\right)^{2}}\right)^{5}}\right], \\
& B_{P y}=\frac{3 \mu_{1} m y}{4 \pi \sqrt{x^{2}+y^{2}}}\left[\frac{\sqrt{(y-h)^{2}+x^{2}}\left(\sqrt{d^{2}-x^{2}}+y \tan \alpha\right)}{\left(\sqrt{(y-h)^{2}+x^{2}+\left(\sqrt{d^{2}-x^{2}}+y \tan \alpha\right)^{2}}\right)^{5}}-\frac{\sqrt{(y+h)^{2}+x^{2}}\left(\sqrt{d^{2}-x^{2}}+y \tan \alpha\right)}{\left(\sqrt{(y+h)^{2}+x^{2}+\left(\sqrt{d^{2}-x^{2}}+y \tan \alpha\right)^{2}}\right)^{5}}\right], \\
& B_{P z}=\frac{\mu_{1} m}{2 \pi}\left[\frac{1-(3 / 2)\left(\left((y-h)^{2}+x^{2}\right) /\left((y-h)^{2}+x^{2}+\left(\sqrt{d^{2}-x^{2}}+y \tan \alpha\right)^{2}\right)\right)}{\left(\sqrt{(y-h)^{2}+x^{2}+\left(\sqrt{d^{2}-x^{2}}+y \tan \alpha\right)^{2}}\right)^{3}}\right.
\end{aligned}
$$

where the intersection angle between $P D$ and the $Z$-axis is $\theta_{2}$ and the distance between point $P$ and point $D$ is $r_{2}$. The geometric relationship in Figure 4 can be expressed as follows:

$$
\begin{aligned}
& r_{1}=\sqrt{(y-h)^{2}+x^{2}+\left(\sqrt{d^{2}-x^{2}}+y \tan \alpha\right)^{2}}, \\
& \theta_{1}=\arctan \frac{\sqrt{(y-h)^{2}+x^{2}}}{\sqrt{d^{2}-x^{2}}+y \tan \alpha}, \\
& r_{2}=\sqrt{(y+h)^{2}+x^{2}+\left(\sqrt{d^{2}-x^{2}}+y \tan \alpha\right)^{2}}, \\
& \theta_{2}=\arctan \frac{\sqrt{(y+h)^{2}+x^{2}}}{\sqrt{d^{2}-x^{2}}+y \tan \alpha}, \\
& \varphi=\arctan \frac{y}{x} .
\end{aligned}
$$

The total magnetic flux density of point $P$ caused by the two magnetic sources can be expressed as 


$$
\left.-\frac{1-(3 / 2)\left(\left((y+h)^{2}+x^{2}\right) /\left((y+h)^{2}+x^{2}+\left(\sqrt{d^{2}-x^{2}}+y \tan \alpha\right)^{2}\right)\right)}{\left(\sqrt{(y+h)^{2}+x^{2}+\left(\sqrt{d^{2}-x^{2}}+y \tan \alpha\right)^{2}}\right)^{3}}\right]
$$

\section{Calculation of the Distance between Adjacent Wells}

The triaxial magnetometer is centered between the two magnetic sources built into the probe tube. The total magnetic flux density detected by the triaxial magnetometer is the sum of the magnetic fields generated by all points of the casing, the two magnetic sources, and the constant geomagnetic field. The triaxial magnetometer detects the magnetic flux density, which is generated by the two magnetic sources and can be expressed as follows:

$$
\begin{aligned}
& B_{C D x}=B_{C x}+B_{D x}=0, \\
& B_{C D y}=B_{C y}+B_{D y}=0, \\
& B_{C D z}=B_{C z}+B_{D z}=0 .
\end{aligned}
$$

The geomagnetic field is usually acquired from international geomagnetic reference field (IGRF) data [8], and it can be regarded as a constant over a short period of time.

The element $P$ of the segmentation casing is far less than the distance between the probe tube and the casing, so it can be regarded as a magnetic dipole (idealized as a point $P$ ).
The magnetic flux density distribution around the magnetic dipole $P$ is derived according to above formulae as follows:

$$
\begin{aligned}
& B_{x}=\frac{3 \mu m^{\prime}}{8 \pi r^{3}} \sin 2 \theta \cos \varphi, \\
& B_{y}=\frac{3 \mu m^{\prime}}{8 \pi r^{3}} \sin 2 \theta \sin \varphi, \\
& B_{z}=\frac{\mu m^{\prime}}{2 \pi r^{3}}\left(1-\frac{3}{2} \sin ^{2} \theta\right),
\end{aligned}
$$

where

$$
\begin{aligned}
& \theta=\arctan \frac{\sqrt{x^{2}+y^{2}}}{\sqrt{d^{2}-x^{2}}+y \tan \alpha}, \\
& r=\sqrt{x^{2}+y^{2}+\left(\sqrt{d^{2}-x^{2}}+y \tan \alpha\right)^{2}}, \\
& \varphi=\arctan \frac{y}{x} .
\end{aligned}
$$

When (17) is substituted into formula (16), the following formulae are deduced:

$$
\begin{aligned}
& B_{x}=\frac{3 \mu m^{\prime} x\left(\sqrt{d^{2}-x^{2}}+y \tan \alpha\right)}{4 \pi\left(\sqrt{x^{2}+y^{2}+\left(\sqrt{d^{2}-x^{2}}+y \tan \alpha\right)^{2}}\right)^{5}}, \\
& B_{y}=\frac{3 \mu m^{\prime} y\left(\sqrt{d^{2}-x^{2}}+y \tan \alpha\right)}{4 \pi\left(\sqrt{x^{2}+y^{2}+\left(\sqrt{d^{2}-x^{2}}+y \tan \alpha\right)^{2}}\right)^{5}}, \\
& B_{z}=\frac{\mu m^{\prime}\left(1-(3 / 2)\left(\left(x^{2}+y^{2}\right) /\left(x^{2}+y^{2}+\left(\sqrt{d^{2}-x^{2}}+y \tan \alpha\right)^{2}\right)\right)\right)}{2 \pi\left(\sqrt{x^{2}+y^{2}+\left(\sqrt{d^{2}-x^{2}}+y \tan \alpha\right)^{2}}\right)^{3}},
\end{aligned}
$$

where the magnetic moment of the magnetic dipole is $m^{\prime}=$ $V \chi_{m} H$. The volume of casing element $P$ is $V$. The magnetic susceptibility of the casing is $\chi_{m}$, and casing diameter is $C$.

$$
m^{\prime}=\frac{V \chi_{m} \sqrt{B_{P x}^{2}+B_{P y}^{2}+B_{P z}^{2}}}{\mu_{1}} .
$$

During the calculation process, the initial magnetic flux density of point $P$ can be calculated by using formula (14). Subsequently, the magnetic moment of the magnetic dipole can be calculated using formula (19). Ultimately, the triaxial magnetometer detects the magnetic flux density of point $P$, which can be calculated using formulae (18). 
In summary, the triaxial magnetometer detects the magnetic flux density of the magnetizing casing, which is composed of a large number of magnetic dipole units, when all the other parameters, except the variable quantity $y$, are all known quantities. Therefore, $d$ can be calculated by reverse deduction, on the condition that the magnetic flux density is known through measurements, using the following:

$$
\begin{aligned}
& B_{\mathrm{Ox}}=\sum_{i=1}^{N} B_{P x i}, \\
& B_{\mathrm{O} y}=\sum_{i=1}^{N} B_{P y i}, \\
& B_{\mathrm{Oz}}=\sum_{i=1}^{N} B_{P z i} .
\end{aligned}
$$

\section{New Segmentation Strategies}

A new variable section segmentation strategy is proposed in Figure 5. It is assumed that the distance between a magnetic dipole unit of the casing model and the measuring point is $d_{n}$. In the new section segmentation, the parameter $l_{n}$, the length of the magnetic dipole unit, is determined by $d_{n}$. The intersection angle between $d_{1}$ and casing axis is $\beta(\beta=\alpha+$ $\pi / 2)$. The relationship between $l_{n}$ and $d_{n}$ can be expressed as [21-25]

$$
\lambda=\frac{d_{n}}{l_{n}}
$$

where $\lambda=10$ is a ratio coefficient [25]. It is ensured that the segmented unit is small enough to be regarded as a magnetic dipole without error.

If there is a measuring point around the casing model, we can determine its horizontal point on the casing. Then, the model is divided from this point; the aim of the segmentation strategy is obtaining the formula of one unit volume $V_{n}$, and it can be expressed as

$$
V_{n}=\pi l_{n} \delta(C-\delta)
$$

To determine $V_{n}$, we need to obtain a formula for the variable $l_{n}$.

First, $l_{1}$ is calculated by $l_{1}=d_{1} / \lambda$ (where $d_{1}=d$ ). Based on the Cosine theorem, we can obtain the following expression:

$$
\left(\frac{l_{1}+l_{2}}{2}\right)^{2}+d_{1}^{2}-\left(l_{1}+l_{2}\right) d_{1} \cos \beta=d_{2}^{2} .
$$

Combined with formulae (21) and (23), the relationship between $l_{1}$ and $l_{2}$ can be deduced using the following:

$$
l_{2}=\frac{\lambda^{2}+1 / 4-\lambda \cos \beta}{\lambda^{2}-1 / 4} l_{1} .
$$

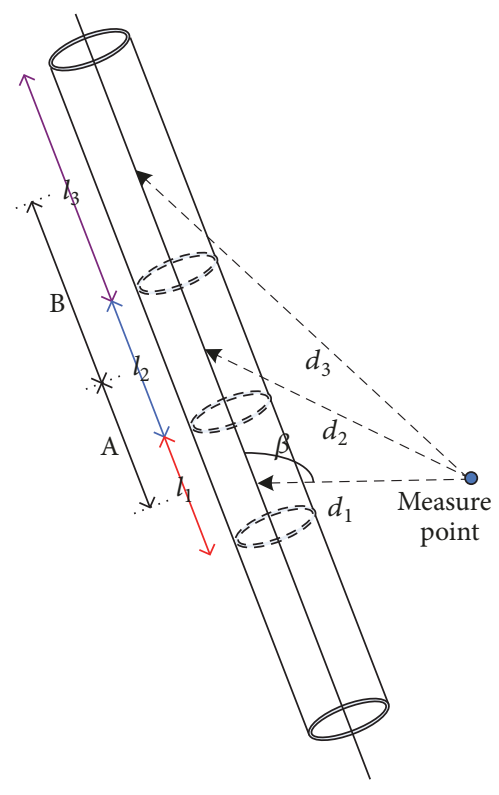

FIGURE 5: The variable section segmentation strategy for the casing.

Second, two representations are shown below:

$$
\begin{aligned}
& B=\frac{l_{n}+l_{n-1}}{2}, \\
& A=\sum_{i=2}^{n-1} \frac{l_{i}+l_{i-1}}{2} .
\end{aligned}
$$

We can also deduce two expressions based on the Cosine theorem:

$$
\begin{aligned}
(B+A)^{2}+d_{1}^{2}-2 d_{1}(B+A) \cos \beta & =d_{n}^{2}, \\
A^{2}+d_{1}^{2}-2 d_{1} A \cos \beta & =d_{n-1}^{2} .
\end{aligned}
$$

Finally, by combining these expressions with formula (21), the expression of $l_{n}$ can be deduced using the following:

$$
l_{n}=\frac{A+\left(\lambda^{2}+1 / 4\right) l_{n-1}-d_{1} \cos \beta}{\lambda^{2}-1 / 4} .
$$

From the expression of $l_{n}$, we can see that the volumes of the divided units in the new segmentation method are different. The unit nearer to the measuring point is smaller, and the unit farther from the measuring point is larger. Expression (27) is substituted into expression (21), and then it can be used to calculate the magnetic moment.

\section{Experimental Results}

During the simulation, it is assumed that $\mu_{0}=4 \pi \times 10^{-7} \mathrm{H} / \mathrm{m}$, $m=10 \mathrm{~A} \cdot \mathrm{m}^{2}, h=1 \mathrm{~m}, \alpha=0^{\circ}, x=0$, and the axes of the probe tube and the casing are on the same plane. As the probe tube works, it turns with a rotation speed of tens to hundreds of revolutions per minute. The drill bit can only advance several millimeters to tens of millimeters per 

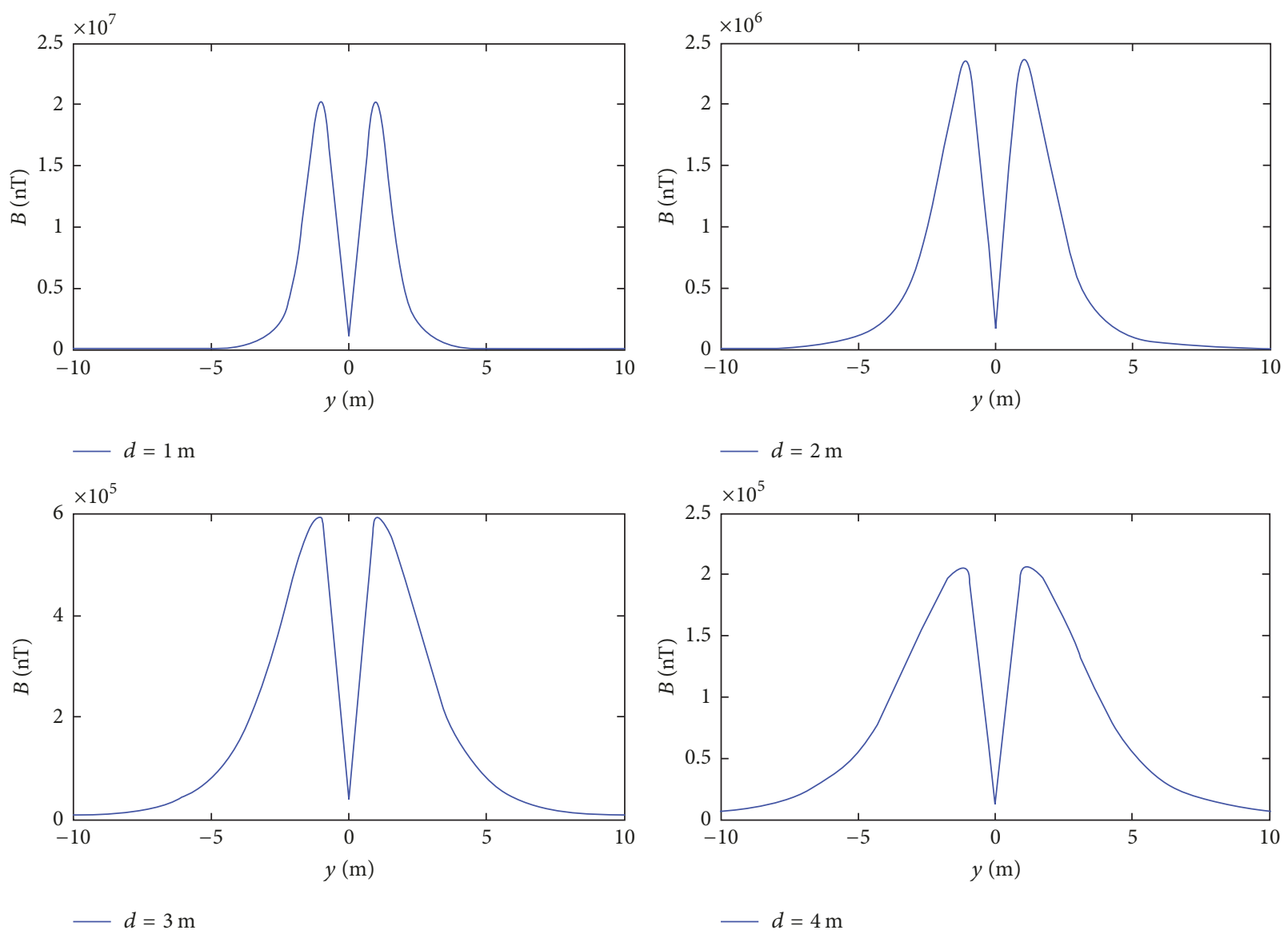

Figure 6: Magnetic flux density simulation for casing.

minute. Compared with the rotation rate, the drilling footage per minute is much smaller. Therefore, it can be assumed that the probe tube stays approximately at the same point relative to adjacent casing when it makes a single turn. The relative distance between probe tube and casing is set to be $1 \mathrm{~m}, 2 \mathrm{~m}, 3 \mathrm{~m}$, or $4 \mathrm{~m}$, and the signal measured by the triaxial magnetometer is simulated by utilizing formula (14). Figure 6 indicates that the triaxial magnetometer detects the strongest magnetic flux density when the axis of the magnetic source points directly to the casing. The magnetic flux density of the casing's magnetic field is of a symmetrical distribution around $y=0$, the peak values of magnetic flux density are mainly concentrated in the vicinity of $y= \pm 1 \mathrm{~m}$, and the magnetic flux density decreases with increasing $y$. Moreover, the magnetic flux density is weaker with increasing distance between the adjacent wells.

\section{Discussion}

Based on the calculations of the active anticollision method, the influence factors of the measurement results mainly include the distance between the two magnetic sources $(2 h)$ in the probe tube, the magnetic moment of the magnetic sources $(m)$, the relative permeability of the casing $\left(\mu_{1}\right)$, the diameter of the casing $(C)$, and the intersection angle $(\alpha)$ between the drilling well and the existing well. It is assumed that the formation is uniformly distributed and isotropic; then, in the absence of any influence from nonferromagnetic minerals in the surrounding stratum, the various influence factors of the measurement results can be analyzed.

7.1. Influence of Magnetic Source on Measurement Results. The magnetic source is the key component of the magnetic anticollision ranging system for adjacent wells; its parameters directly affect the range and accuracy of the measured distances. The main parameters that influence the measurement accuracy include the distance $(2 h)$ between the two magnetic sources and the magnetic moment $(m)$ of the magnetic sources. By choosing $\mu_{0}=4 \pi \times 10^{-7} \mathrm{H} / \mathrm{m}, \mu_{1}=1000, \alpha=0^{\circ}$, $C=127 \mathrm{~mm}, m=10 \mathrm{~A} \cdot \mathrm{m}^{2}$, and different values of $2 h$, the measurement results shown in Figure 7 indicate that the distance between the casing and the probe tube vary in the range of $0.4 \sim 2 \mathrm{~m}$.

Figure 7 illustrates that magnetic flux density of the probe tube detection increases with increasing $2 h$ when the magnetic moment is invariant. However, its variability is small and may be ignored under the condition of $2 h>$ $1.2 \mathrm{~m}$. Therefore, considering the length of the probe tube itself, the maximum magnetic flux density of the probe tube is observed under the condition of $2 h=1.2 \mathrm{~m}$. Influence of 


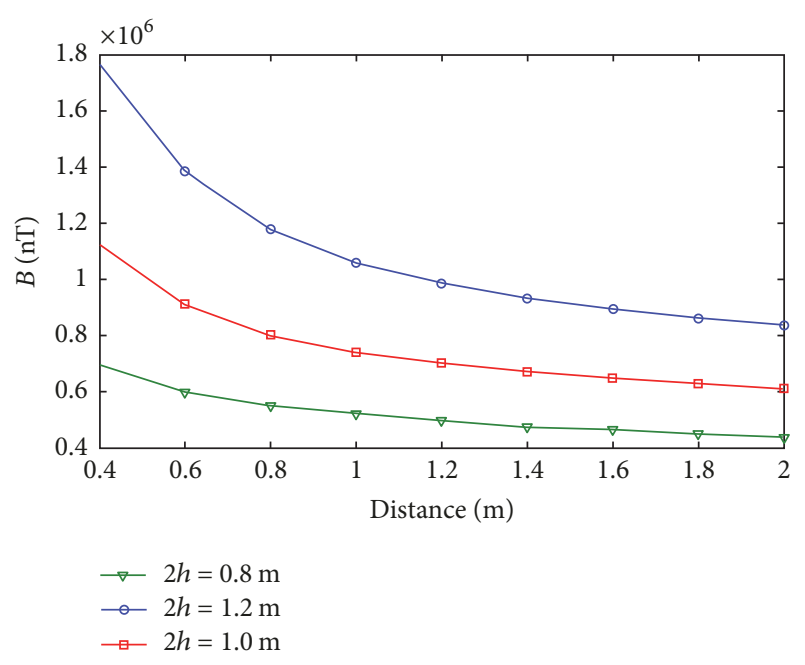

Figure 7: Influence of the distance between double magnetic sources on measurement results.

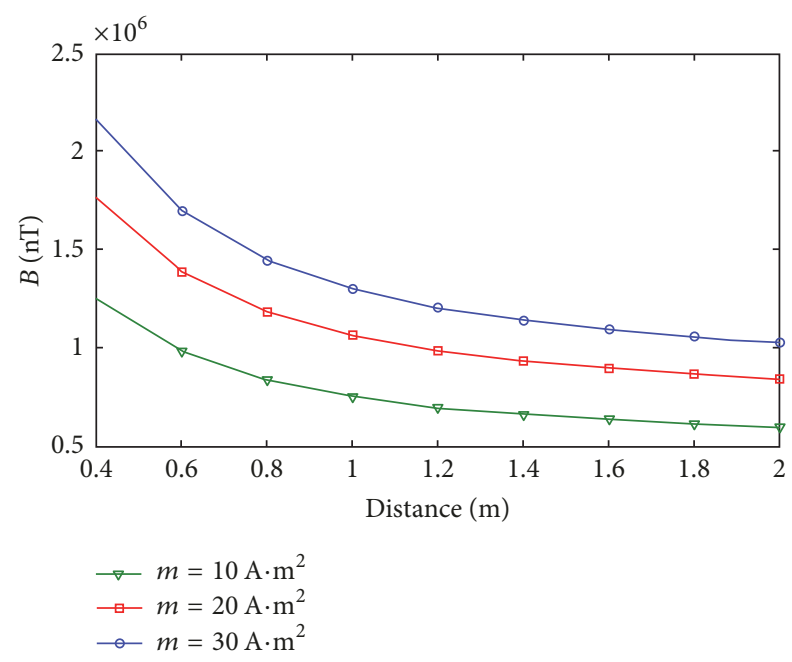

FIGURE 8: Influence of magnetic moment on measurement results.

the different values of the magnetic moment of the magnetic sources on the magnetic flux density measurement results is shown in Figure 8 for $2 h=1.2 \mathrm{~m}$. Figure 8 indicates that the magnetic flux density detected by the probe tube increases with increasing magnetic moment when $2 h$ is invariant. Therefore, we should choose magnetic sources with large magnetic moments when designing the probe tube's structure.

7.2. Influence of the Casing on Measurement Results. The strata surrounding the probe tube are less affected by the magnetic flux density generated by the magnetic sources, but the adjacent well's casing with high permeability is greatly affected by the magnetic flux density generated by the magnetic sources. Supposing $2 h=1.2 \mathrm{~m}, \alpha=0^{\circ}, C=127 \mathrm{~mm}$, $m=10 \mathrm{~A} \cdot \mathrm{m}^{2}$, and different values of $\mu_{1}$, the measurement results are produced as shown in Figure 9, in which the

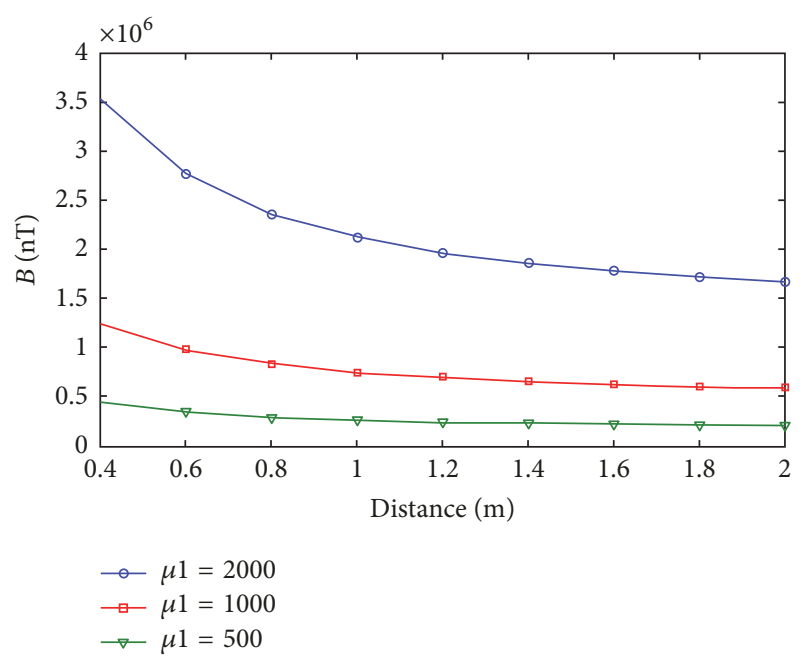

FIGURE 9: Influence of the relative magnetic conductivity of the casing on measurement results.

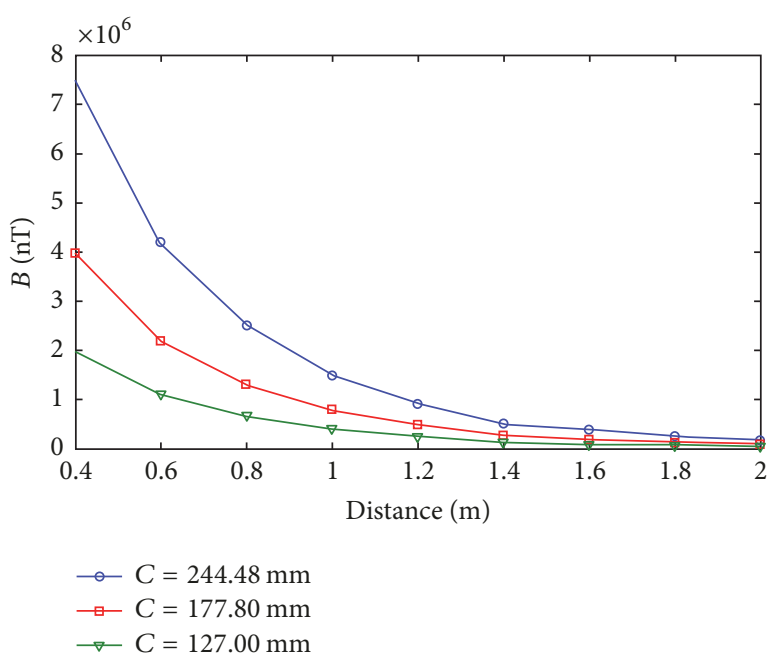

FIGURE 10: Influence of the diameter of casing on measurement results.

distance between the casing and the probe tube ranges within $0.4 \sim 2 \mathrm{~m}$.

Figure 9 illustrates that the magnetic flux density detected by the probe tube increases with the increasing the relative permeability of the casing when the parameters of the magnetic source are invariant. The relative permeability of the casing is determined by the casing's properties. Therefore, if the relative permeability of the adjacent casing is measured before calculating the distance between the adjacent wells, then a more accurate relative permeability can be obtained to ensure the accuracy of the magnetic anticollision ranging system.

When the relative permeability of the casing is $\mu_{1}=1000$, different values are used for the diameter of the casing $(C)$ and the distance $(d)$ between the casing, and the probe tube ranges within $0.4 \sim 2 \mathrm{~m}$, the measurement results of the simulation are shown in Figure 10. 


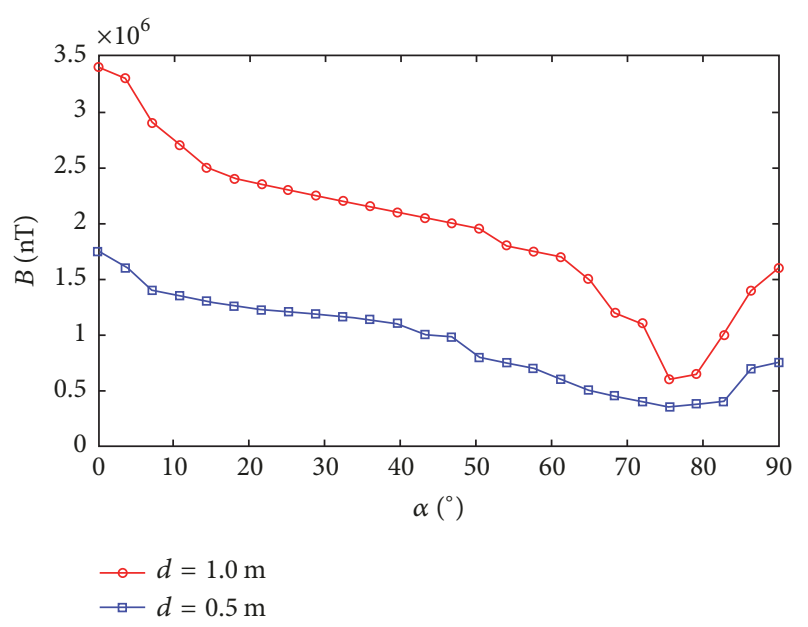

FIGURE 11: Influence of the intersection angle $\alpha$ on measurement results.

Figure 10 indicates that the magnetic flux density detected by the probe tube increases as the diameter of the casing increases when the relative permeability of the casing is invariant, and the risk of colliding with the larger diameter casing is increased under the same distance between adjacent wells.

7.3. Influence of the Intersection Angle $\alpha$ on Measurement Results. In the cluster wells, there is an intersection angle $(\alpha)$ between the drilling well and the target well. Assuming that $2 h=1.2 \mathrm{~m}, m=10 \mathrm{~A} \cdot \mathrm{m}^{2}, \mu_{1}=1000$, and $C=127.0 \mathrm{~mm}$, when $\alpha$ changes from $0^{\circ}$ to $90^{\circ}$, the distance between the casing and the probe tube ranges within $0.4 \sim 2 \mathrm{~m}$ within the measurement results simulation, which produces the results shown in Figure 11.

Figure 11 indicates that the change in the magnetic flux density detected by the probe tube is reduced to half of the original value when the intersection angle $\alpha$ changes from $0^{\circ}$ to $50^{\circ}$. In addition, there is no correlation between the magnetic flux density and $\alpha$ when $\alpha>50^{\circ}$. According to the analysis, this is because when the angle between the drilling well and the adjacent well is too large, the magnetic field generated by the magnetic source farther from the casing within the probe is too weak at the casing. It is equivalent to the scenario in which only one magnetic source in the probe tube generates the magnetization of the casing, and the distribution of the flux density changes, resulting in the distortion of the curve. Therefore, it is necessary to avoid the application of this method under conditions with $\alpha>50^{\circ}$.

\section{Conclusions}

The magnetic anticollision system for cluster well is a kind of magnetic guidance tool that can determine the measurement distance while drilling in real time. The principle of the method is based on detecting the magnetic flux density of the magnetizing casing, in which the two magnetic sources are parallel to each other with opposite magnetic fields and installed at two ends of the built-in tube probe. First, with the rotation of the probe tube, the triaxial magnetometer detects the oscillation of the signal from the casing. Then, the signal is transmitted to the ground by the mud pulse. Ultimately, the distances and azimuth of adjacent wells are calculated by data analysis software.

The radii of the magnetic sources are far less than the borehole distance, so the magnetic sources can be regarded as magnetic dipoles. The magnetic flux density distribution formulae of the magnetic dipole are deduced by the magnetic flux density calculation method for a magnetic dipole. The simulation results indicate that a triaxial magnetometer can detect the maximum or minimum value of the magnetic field intensity when the axis of the magnetic source points directly to the casing.

The magnetic flux density detected by the probe tube increases with increasing distance between the double magnetic sources; when its distances exceeds $1.2 \mathrm{~m}$, there is no significant change in the magnetic flux density detected. The magnetic flux density of the probe is positively related to the magnetic moment of the magnetic sources, the relative permeability of the casing, and the diameter of the adjacent well casing pipe, so that it can reach farther measuring distances and higher ranging accuracies by increasing these parameters' values. Accurate measurement results can be obtained by the method of adjacent well location under the condition of $\alpha<50^{\circ}$. This method can provide theoretical support and an experimental basis for the development of the active magnetic guidance ranging for cluster wells.

\section{Data Availability}

The data used to support the findings of this study are available from the corresponding author upon request.

\section{Conflicts of Interest}

The authors declare that they have no conflicts of interest.

\section{Acknowledgments}

The authors gratefully acknowledge the financial support of National Science and Technology Major Project "New Technologies of Designing and Controlling for the Complex Structure Wells and the Cluster Wells (Grant no. 2017ZX05009-003)".

\section{References}

[1] D. L. Gao and B. B. Diao, "Development of the magnetic guidance drilling technique in complex well engineering," Petroleum drilling techniques, vol. 44, no. 5, pp. 1-9, 2016.

[2] D. Gao, B. Diao, Z. Wu, and Y. Zhu, "Research into magnetic guidance technology for directional drilling in SAGD horizontal wells," Petroleum Science, vol. 10, no. 4, pp. 500-506, 2013.

[3] A.-M. Al-Bahlani and T. Babadagli, "SAGD laboratory experimental and numerical simulation studies: A review of current status and future issues," Journal of Petroleum Science and Engineering, vol. 68, no. 3-4, pp. 135-150, 2009. 
[4] D. A. Wood, "Drilling and borehole techniques relevant to natural gas exploration and development: A collection of published research (2009-2015)," Journal of Natural Gas Science and Engineering, vol. 26, pp. 396-408, 2015.

[5] B. Diao and D. Gao, "A magnet ranging calculation method for steerable drilling in build-up sections of twin parallel horizontal wells," Journal of Natural Gas Science and Engineering, vol. 27, pp. 1702-1709, 2015.

[6] B. Diao and D. Gao, "Study on a ranging system based on dual solenoid assemblies, for determining the relative position of two adjacent wells," CMES: Computer Modeling in Engineering \& Sciences, vol. 90, no. 1, pp. 77-90, 2013.

[7] B. B. Diao, D. L. Gao, and Z. Y. Wu, "Magnet ranging calculation method of twin parallel horizontal wells steerable drilling," Journal of China university of petroleum, vol. 35, no. 6, pp. 71$75,2011$.

[8] C. C. Finlay, S. Maus, C. D. Beggan et al., "International Geomagnetic Reference Field: The eleventh generation," Geophysical Journal International, vol. 183, no. 3, pp. 1216-1230, 2010.

[9] Z. Guan, Y. Liu, and Y. Shi, "Problems and developing direction of anti-collision technology in the dense well pattern area," in Proceedings of the Procedia engineering, vol. 7, pp. 304-311, 2010.

[10] T. Liu and B. Wang, "Study of magnetic ranging technology in horizontal directional drilling," Sensors and Actuators A: Physical, vol. 171, no. 2, pp. 186-190, 2011.

[11] G. Liu, Q. Yang, Z. Dong, B. He, and Z. Geng, "A drill bit vibration anti-collision monitoring system and field experiment," Natural Gas Industry, vol. 33, no. 6, pp. 66-70, 2013.

[12] M. Marchetti, V. Sapia, and A. Settimi, "Magnetic anomalies of steel drums: A review of the literature and research results of the INGV," Annals of Geophysics, vol. 56, no. 1, 2013.

[13] S. D. Billings, C. Pasion, S. Walker, and L. Beran, "Magnetic models of unexploded ordnance," IEEE Transactions on Geoscience and Remote Sensing, vol. 44, no. 8, pp. 2115-2123, 2006.

[14] Z. Wu, D. Gao, and B. Diao, "An investigation of electromagnetic anti-collision real-time measurement for drilling cluster wells," Journal of Natural Gas Science and Engineering, vol. 23, pp. 346-355, 2015.

[15] W. Yang, C. Hu, M. Li, M. Q.-H. Meng, and S. Song, "A new tracking system for three magnetic objectives," IEEE Transactions on Magnetics, vol. 46, no. 12, pp. 4023-4029, 2010.

[16] Z. Y. Zhang, C. H. Xiao, W. Chen, and G. Zhou, "Experiment research of magnetic dipole model applicability for a magnetic object," Journal of basic Science and engineering, vol. 18, no. 5, pp. 862-868, 2010.

[17] J. Simpson, J. Lane, C. Immer, and R. Youngquist, "Simple analytic expressions for the magnetic field of a circular current loop," NASA technical document collection document, pp. 1-3, 2001.

[18] V. Sanchez, L. Yaoguo, M. N. Nabighian, and D. L. Wright, "Numerical modeling of higher order magnetic moments in UXO discrimination," IEEE Transactions on Geoscience and Remote Sensing, vol. 46, no. 9, pp. 2568-2583, 2008.

[19] B. Tu, Li. DS, and EH. Lin, "Analysis of drilling parallel horizontal twin wells rotating magnetic beacons magnetic field strength size in SAGD," in Proceedings of the PIERS Proceedings, vol. 7, pp. 5-8, 2010.

[20] D. Wang and D. Gao, "Study of magnetic vector guide system in tubular magnet source space," Shiyou Xuebao/Acta Petrolei Sinica, vol. 29, no. 4, pp. 608-611, 2008.
[21] S. Feng, D. Liu, X. Cheng, H. Fang, and C. Li, "A new segmentation strategy for processing magnetic anomaly detection data of shallow depth ferromagnetic pipeline," Journal of Applied Geophysics, vol. 139, pp. 65-72, 2017.

[22] Z. Guo, D. Liu, and Y. Luo, "Error analysis of magnetic field calculation using magnetic dipole based on circular current," International Journal of Applied Mathematics and Statistics, vol. 51, no. 24, pp. 121-130, 2013.

[23] Z.-Y. Guo, D.-J. Liu, Q. Pan, and Y.-Y. Zhang, "Forward modeling of total magnetic anomaly over a pseudo-2D underground ferromagnetic pipeline," Journal of Applied Geophysics, vol. 113, pp. 14-30, 2015.

[24] Z. Guo, D. Liu, Q. Pan, Y. Zhang, Y. Li, and Z. Wang, "Vertical magnetic field and its analytic signal applicability in oil field underground pipeline detection," Journal of Geophysics and Engineering, vol. 12, no. 3, article no. 340, pp. 340-350, 2015.

[25] Q. Pan, D.-J. Liu, Z.-Y. Guo, H.-F. Fang, and M.-Q. Feng, "Magnetic anomaly inversion using magnetic dipole reconstruction based on the pipeline section segmentation method," Journal of Geophysics and Engineering, vol. 13, no. 3, pp. 242-258, 2016. 


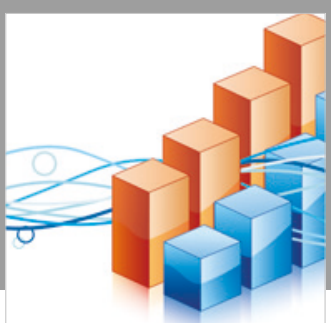

Advances in

Operations Research

\section{-n-m}
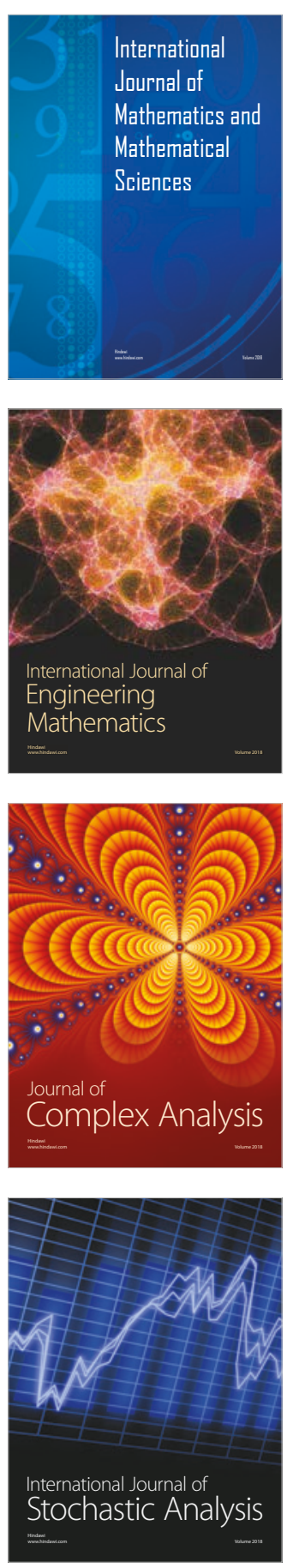
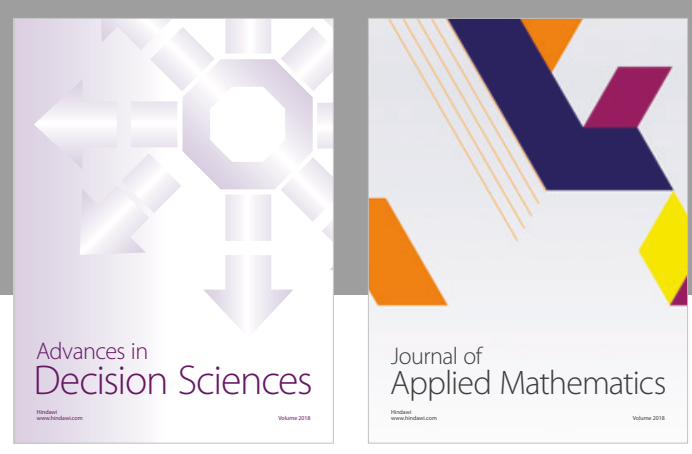

Journal of

Applied Mathematics
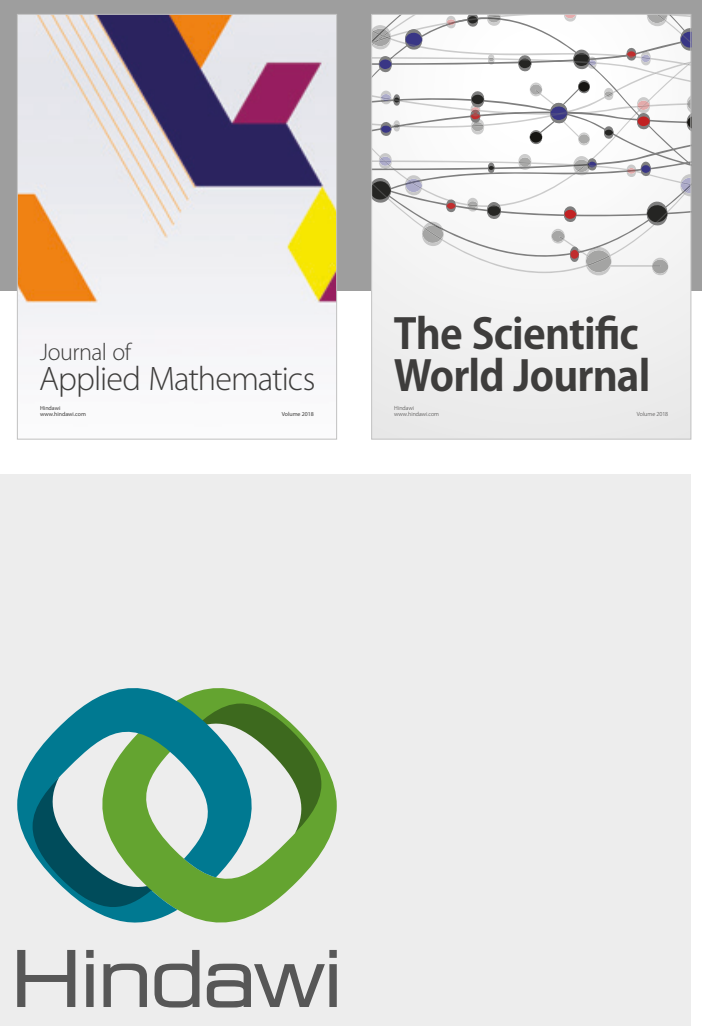

Submit your manuscripts at

www.hindawi.com

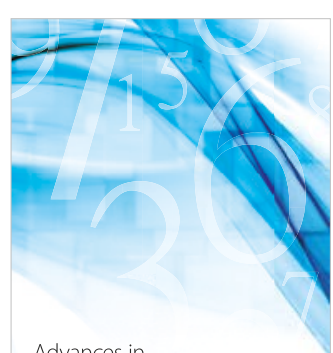

Advances in
Numerical Analysis
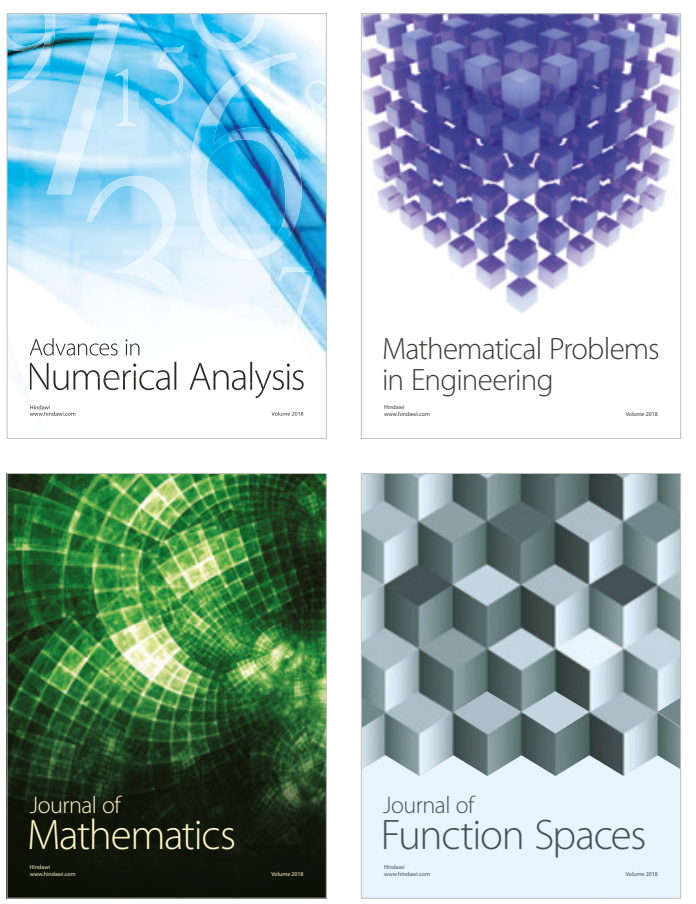

Mathematical Problems in Engineering

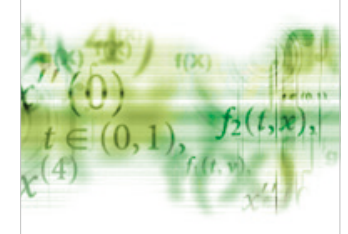

International Journal of

Differential Equations

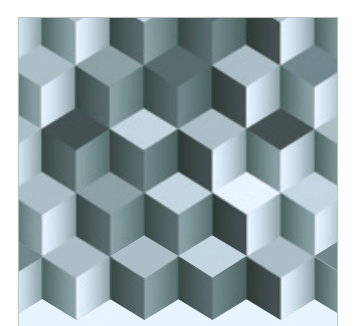

Journal of

Function Spaces

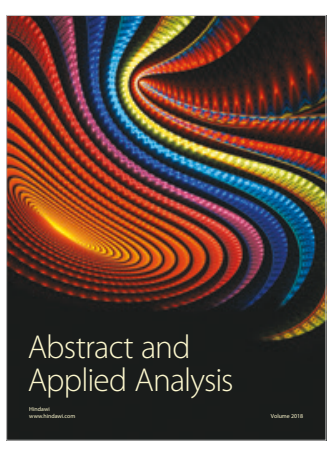

The Scientific

World Journal

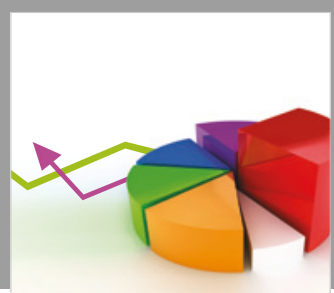

Journal of

Probability and Statistics
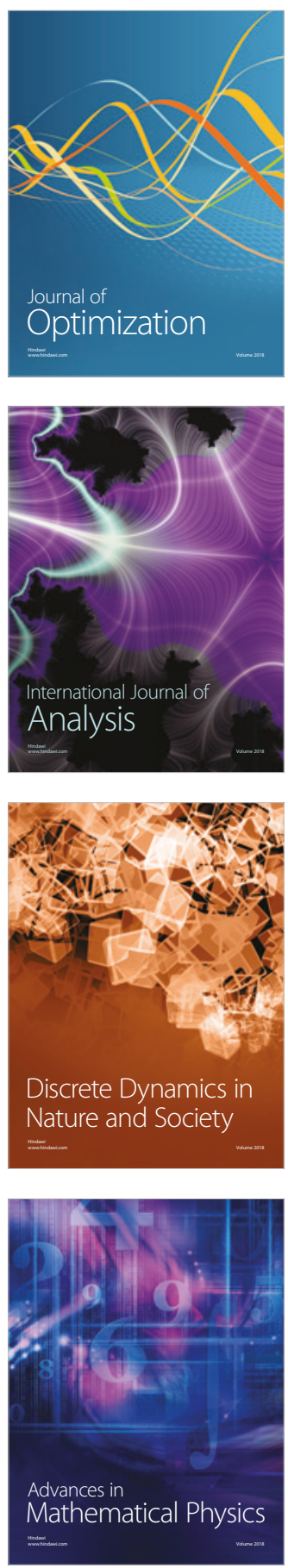